\title{
Release of RANKERN 16A
}

\author{
Adam Bird ${ }^{1, *}$, Christophe Murphy ${ }^{1}$, and Geoff Dobson $^{1}$ \\ ${ }^{1}$ AMEC Foster Wheeler, King's Point House, Poundbury, Dorchester, Dorset DT1 3BW, UK
}

\begin{abstract}
RANKERN 16 is the latest version of the point-kernel gamma radiation transport Monte Carlo code from AMEC Foster Wheeler's ANSWERS Software Service. RANKERN is well established in the UK shielding community for radiation shielding and dosimetry assessments. Many important developments have been made available to users in this latest release of RANKERN. The existing general 3D geometry capability has been extended to include import of CAD files in the IGES format providing efficient full CAD modelling capability without geometric approximation. Import of tetrahedral mesh and polygon surface formats has also been provided. An efficient voxel geometry type has been added suitable for representing CT data. There have been numerous input syntax enhancements and an extended actinide gamma source library. This paper describes some of the new features and compares the performance of the new geometry capabilities.
\end{abstract}

\section{Introduction}

RANKERN is a well-established and powerful pointkernel Monte Carlo software tool for general gamma-ray radiation transport analysis for shielding and dosimetry applications. RANKERN is developed and licensed by AMEC Foster Wheeler's ANSWERS Software Service. The RANKERN package comprises not only the code itself but also nuclear data libraries, user documentation, productivity tools and user support services. Supporting geometry model visualisation and verification and results visualisation tools are also available.

The uncollided flux estimates produced by RANKERN are corrected for scattering using build-up factors; three build-up options are available. The first, known as the original method, expresses the build-up data in the form of cubic polynomials. The coefficients can be supplied directly or included from library data derived from a combination of reference's [1] and [2] and MCBEND[3] Monte Carlo simulations. In addition a method is available to estimate the importance of oblique build-up. The second method is a set of build-up libraries based on ANS-6.4.3 [4] recommendations. These extend the number of materials and penetration distances from 20 to $40 \mathrm{mfp}$ and are the current default in RANKERN. The third option uses Diffusion Theory to calculate build-up factors. This is particularly useful for multi-layered shields without a dominant final shield material.

RANKERN is being continually developed to meet the needs of its users. RANKERN 16 is the latest version to be released in line with the ANSWERS vision to 'provide easy-to-use software that meets the current and emerging needs of the user community'. In the case of RANKERN 16 this vision focuses on the key areas of geometry modelling and user-friendliness. This paper details the developments incorporated into RANKERN 16 and some associated capabilities of the accompanying graphics software VisualWorkshop[5]. All the images in this paper were generated using VisualWorkshop.

\section{Developments in RANKERN 16}

\subsection{Geometry Modelling}

The geometry modelling system employed by RANKERN is known as Fractal Geometry (FG). FG is a well-established system of solid geometry modelling in which the problem geometry is subdivided into zones defined as the intersections and differences of mathematical bodies. RANKERN has a large selection of body shapes ranging from simple bodies such as cuboids, cylinders and spheres to more complex bodies such as prisms, ellipses and tori. The bodies are assembled into structures called parts, which are self contained with their own local co-ordinate system to simplify the model construction. Parts may be included within other parts to any depth of nesting and a given part may be included more than once within the geometry. The ability to break down complex models into parts simplifies the preparation and checking of the input data. The FG geometry package is shared with the ANSWERS radiation transport Monte Carlo code MCBEND and the ANSWERS Criticality code MONK $^{\circledR}[6]$. RANKERN 16 benefits from improvements to geometry checking and the addition of two simple geometric bodies, a triangular prism and a rod with an elliptical cross section. The main enhancements come in the form of CAD geometry import capabilities which are detailed below.

Corresponding author: adam.bird@amecfw.com

C) The Authors, published by EDP Sciences. This is an open access article distributed under the terms of the Creative Commons Attribution License 4.0 (http://creativecommons.org/licenses/by/4.0/). 


\subsubsection{Import of a CAD file in the IGES format}

This is a fully featured CAD import capability that provides particle tracking within the CAD model with no conversion or loss of accuracy of the geometry of the model. The interactive display within VisualWorkshop identifies the figures and sub-figure definitions of the IGES model making it simple to assign material compositions to regions of space within the CAD model. See Figure 1 for an example.

\subsubsection{Import of a tetrahedral mesh format file}

While there is little reason to convert a file to a tetrahedral mesh format for the sole purpose of importing into RANKERN it is quite common for these files to exist having been generated for a stress analysis or thermodynamics physics code for example. In this case gamma shielding assessments can be performed without the need to re-create the geometry model. Groups of tetrahedra that represent a named item within the mesh model are automatically assigned to a material of the same name in RANKERN. See Figure 2 for an example.

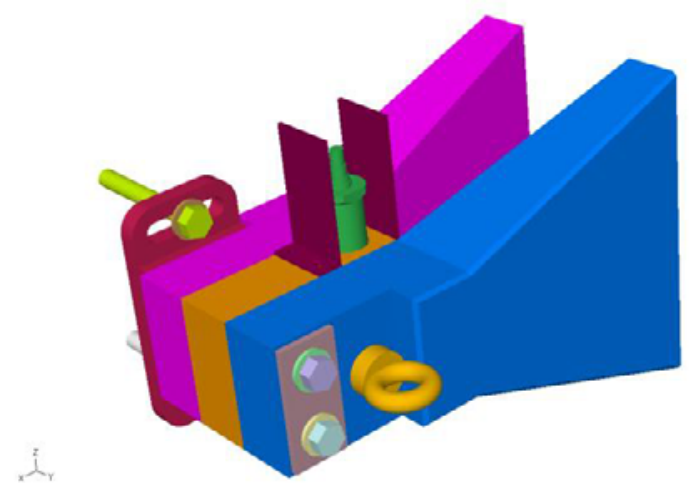

Figure 1. An example IGES model displayed by the 3D ray trace feature of VisualWorkshop .

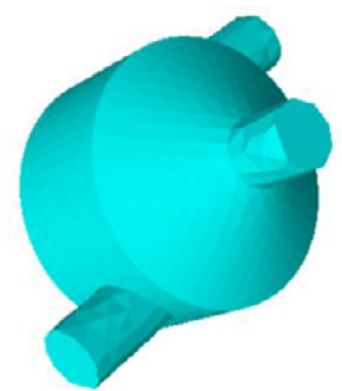

Figure 2. An example tetrahedral mesh model.

\subsubsection{Import of a polygon surface format file}

A triangular polygon surface geometry type can be imported or defined directly in the RANKERN input as a native FG body type.

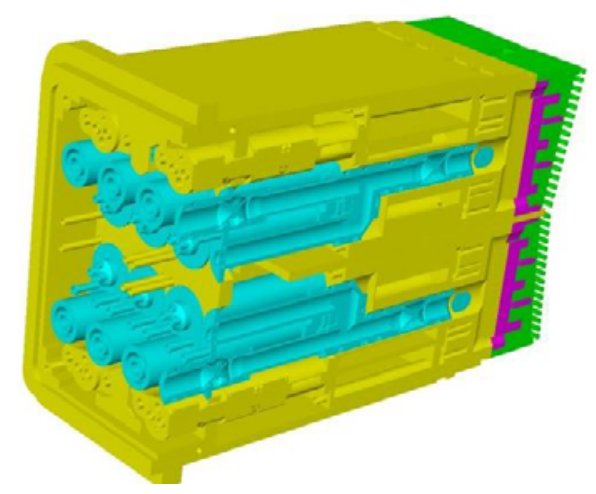

Figure 3. An example model that has been exported from a CAD package in a polygon surface format.

Many CAD packages have options for exporting a polygon surface description and this is usually faster to process than the IGES import and requires less memory than a tetrahedral mesh representation.

\subsubsection{Voxel geometry representation}

This geometry type can be imported or defined directly in the RANKERN input. A voxel geometry could be represented by many boxes. The voxel geometry type in RANKERN takes advantage of the regularity of the geometry to reduce memory requirements and provide efficient tracking.

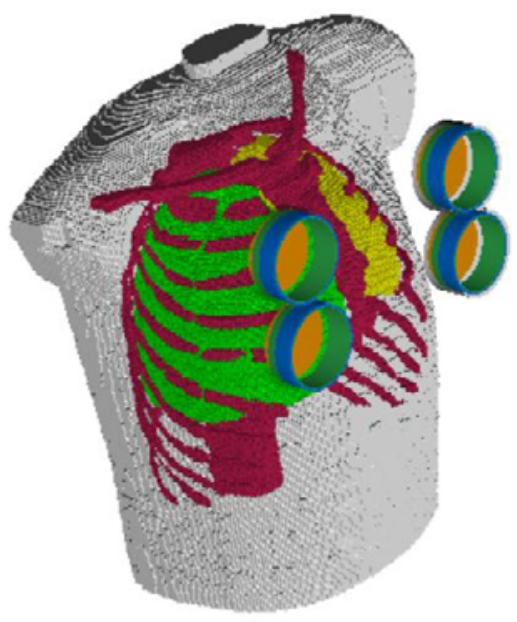

Figure 4. An example of a voxel geometry model from CT data with detectors defined using FG.

All of the geometry types described can be used in a mix and match way in a given model. Section 4 reports on the relative performance of the geometry methods.

\subsection{Visualisation}

VisualWorkshop version 3B, which accompanies RANKERN, includes some enhancements to contour plotting of results from RANKERN and can now display the particle tracks from a RANKERN calculation. 


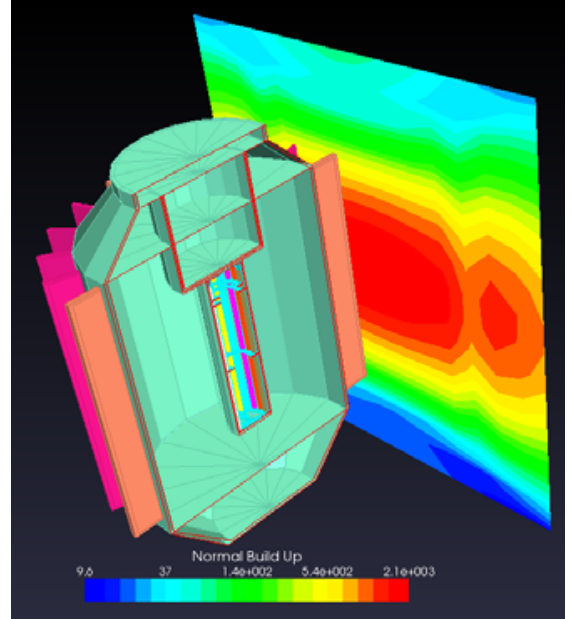

Figure 5. VisualWorkshop image showing a dose rate contour generated from a grid of RANKERN output points.

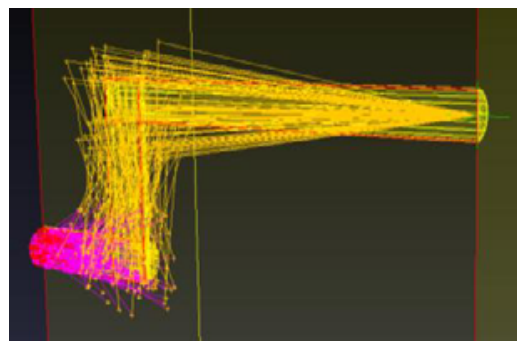

Figure 6. VisualWorkshop image showing RANKERN tracks through a duct.

\section{Other enhancements}

\subsection{RKARD Package Enhancements}

The RKARD system of data input provides a powerful method of using mathematical and logical functions to construct a flexible and efficient input model.

RANKERN 16 has enhanced RKARD facilities including:

- Double precision arithmetic to increase precision

- Exponentiation to any power

- Functions INT, ATAN, ACOS and ASIN

- Variables containing character string values

- Variables containing logical conditions

- Parameters used for looping containing variables

- Nested IF statement blocks

- ELSEIF statements

- Loop counters in conditions

- Extended condition statements

- Additional RKARD controls

\subsection{Source Enhancements}

Sources in RANKERN can be defined in terms of an energy group source spectrum or as an inventory of actinides. The latter can optionally be decayed before the calculation commences. For this release the source and decay libraries have been extended and now contain data for over 1800 actinides.

\subsection{Looping}

The input data for RANKERN can contain variables. Variables can be given lists of values and a calculation is run for each one. This is called a 'looping' calculation. A new command line argument specifying the loop index is available to select one of the calculations in a RANKERN looping case to run. This makes it possible to run RANKERN looping cases in parallel on a computing cluster.

\subsection{New Random Number Generator}

RANKERN now uses the same random number generator as the MCBEND and MONK codes. In previous versions of RANKERN if a seed was not provided then the same default seed was used each time. In RANKERN 16 if a seed is not provided it will be generated from the date and time of execution.

\section{Performance implications of the geometry representation chosen}

The native 3D Geometry capabilities of RANKERN are powerful and general and enable complex models to be constructed relatively easily. However, they need to be created by the modeller. Importing from $3^{\text {rd }}$ party CAD software can save a user time. The relative performance of the imported geometry is important because the CAD geometry is not converted. As a result there are no geometric approximations and this comes with increased computational requirements.

Fractal Geometry, IGES, Polygon Surface and Tetrahedral Mesh versions of the model shown in Figure 5 were created and the relative computing time was compared for each one - see Table 1 .

Table 1. Relative computing time for each geometry type.

\begin{tabular}{|c|c|}
\hline Geometry Type & Relative Speed \\
\hline Fractal Geometry & 1.0 \\
\hline IGES & 3.4 \\
\hline Polygon Surface & 3.9 \\
\hline Tetrahedral Mesh & 6.4 \\
\hline
\end{tabular}

Thus there is a trade-off between ease of model preparation and run time. This is only likely to be an issue for very complex models with a lot of dose points or if a large number of calculations need to be performed. 


\section{Conclusions}

This paper has summarised the developments incorporated into RANKERN 16. These cover improvements to the geometry modelling with the introduction of CAD import features and improvements to the RKARD input system amongst others. ANSWERS continues to be committed to meeting the current and future needs of its customers.

\section{References}

1. M Capo. Ray build-up factors for point polynomial approximation of gamma isotropic sources, APEX 510

2. J. N. Stone and H. H. Vav Tuyl, PUSHLD - A code for calculating gamma dose rates for plutonium in various geometries. HEDL-TME 73-89.

3. P. Cowan, G. Dobson, J. Martin, Release of MCBEND11, ICRS12-RPSD2012, Nara, Japan.

4. RSIC Data Library Collection. ANS-6.4.3 Geometric progression gamma-ray build-up factor coefficients, 1991. DLC-129

5. A. Bird and T. Fry, Visual Workshop 2: A Model Viewer, Editor and Results Display Package for the Answers Shielding and Criticality Codes, ICRS12RPSD2012, Nara, Japan.

6. S. Richards, D. Long and P. Smith MONK10: $A$ Monte Carlo Code for Criticality Analysis., ICNC2015, Charlotte, NC, USA 